\title{
NOISE ONBOARD 9000 CBM OIL TANKER
}

\author{
Bosoanca Ioan \\ "Dunarea de Jos" University of Galati, \\ Faculty of Naval Architecture, Galati, Domneasca \\ Street, No. 47, 800008, Romania, \\ E-mail:ioan.bosoanca@ugal.ro
}

\begin{abstract}
The paper shows the noise levels in cabins or work places for seafarers. The measured values are compared with the Marine Safety Committee acceptable limits, revised by MSC 337(91) CODE ON NOISE LEVELS ONBOARD SHIP for a better acoustic comfort, both for crew and passengers.

The results obtained from measurements in sea trials, showed some consistent exceeding of the limit values in certain spaces which could be avoided if a noise prediction for this ship would be provided early in design stage of vessels.
\end{abstract}

Keywords: Noise, pollution factor, Code on noise levels onboard ships, acoustic comfort, noise prediction

\section{INTRODUCTION}

Noise is an important pollution factor onboard ships, affecting both crew and passengers.

Why has noise become so important in these days? A lot of papers have shown the adverse effects of noise:

- Noise hearing: the hearing threshold can increase irremediable and the human subject can lose the perception of noise, if a stimulus greater than $80 \mathrm{~dB}(\mathrm{~A})$ acts a longer time (more than 8 hours / day);

- $\quad$ Sleeping problems: the sleep is very important for human being, because during sleep process the organism recovers the physical and psyche force including work capacity;
- Noise can induce hart diseases by vasoconstriction effect;

- Affects communication with others persons and can induce auto isolation;

- Increases the gastric secretion. An increase of the gastric juice can induce ulcer.

These are just some of hazardous effects of high noise exposure levels on a longer period of time.

Therefore, the latest revision of CODE ON NOISE LEVELS ONBOARD SHIP, MSC 337(91) [1] has been released. The new version of the Marine Safety Committee (MSC) introduced a noise level decreasing with 5 $\mathrm{dB}(\mathrm{A})$ for certain spaces, onboard commercial and passenger ships; the new values have been adopted instantly by all the classification societies and became mandatory. 


\section{9K CBM OIL TANKER}

This ship has twin screw, oil/chemical tanker, having a capacity of $9000 \mathrm{dwt}$.

The ship is:

- diesel mechanic driven;

- single deck;

- double hull;

- with 2 CPP propeller / 4 blades / 3.0 m diameter.

The vessel is suitable for transport of oil and chemical products having a flash point of bellow $60^{\circ} \mathrm{C}, 5$ segregations with double valves.

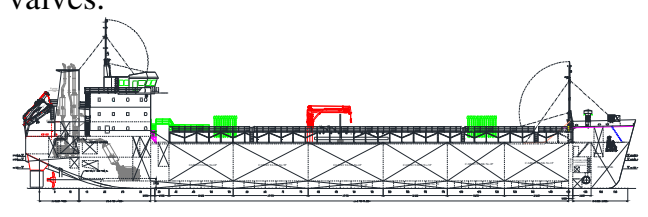

Fig. 2.1 Lateral view of the ship

\section{MAIN PARTICULARS:}

\section{Hull:}

- length overall approx. $119.5 \mathrm{~m}$;

- length between p.p. approx. $115.32 \mathrm{~m}$;

- breadth molded approx. $21.6 \mathrm{~m}$;

- depth approx. $8.64 \mathrm{~m}$;

- design draft $6.2 \mathrm{~m}$;

- deadweight 9000 dwt.

\section{Machinery:}

- 2 main engines $2040 \mathrm{~kW} \mathrm{/} 1000 \mathrm{rpm}$ at MCR;

- 2 diesel generator $860 \mathrm{~kW} \mathrm{/} 1000 \mathrm{rpm}$ fueled with marine gas oil (MGO);

- 2 CPP with 4 blades at $224.7 \mathrm{rpm}$; propeller diameter $=3.00 \mathrm{~m}$;

- 2 shaft generators of $861 \mathrm{~kW}$.

\section{TRIALS CONDITIONS}

The noise measurements have been performed on the Black Sea (Constantza harbor area), in sea trials, after endurance tests have been completed.

Draught at trials: $\mathrm{T}_{\text {fore }}=\mathrm{T}_{\mathrm{aft}}=\mathrm{Tm}=6.2 \mathrm{~m}$ (full load condition).

Weather conditions:

- sea: $2^{\mathrm{O}} \mathrm{D}$;

- wind force: $3^{\mathrm{O}} \mathrm{B}$;

- water temperature: $17^{\mathrm{O}} \mathrm{C}$.

Water depth was more than 5 times under keel draught (minimum depth $=$ ab. $34 \mathrm{~m}$ ).

The results are shown in the bellow table, in accordance with [2]:

\section{Table 3.1 Measuring results}

\begin{tabular}{|c|c|c|c|c|c|}
\hline \multicolumn{4}{|c|}{ Measuring point } & \multirow{2}{*}{$\begin{array}{l}\text { Reading } \\
\text { Leq[dB } \\
\text { (A)] }\end{array}$} & \multirow{2}{*}{$\begin{array}{l}\text { Limit } \\
\text { Leq } \\
\text { [dB (A) }]\end{array}$} \\
\hline $\begin{array}{l}\text { Pos- } \\
\text { ition }\end{array}$ & Room & $\begin{array}{l}\text { ․ } \\
0\end{array}$ & 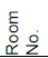 & & \\
\hline 1. & Wingbridge PS & Bridge & & 71.5 & 70 \\
\hline 2. & Wheelhouse PS & Bridge & & 60.2 & 65 \\
\hline 3. & Wheelhouse SB & Bridge & & 60.7 & 65 \\
\hline 4. & Wingbridge SB & Bridge & & 68.4 & 70 \\
\hline 5. & AC room & Bridge & & 80.2 & 95 \\
\hline 6. & Chart room & Bridge & & 63.5 & 65 \\
\hline 7. & Ch. Eng. living & B & & 57.4 & 60 \\
\hline 8. & Ch. Eng. bedroom & B & & 56.7 & 60 \\
\hline 9. & $2^{\text {nd }}$ Eng. & B & & 57.1 & 60 \\
\hline 10. & $2^{\text {nd }}$ Off. & B & & 57.8 & 60 \\
\hline 11. & Ch. Off. living & B & & 63.1 & 60 \\
\hline 12. & Ch. Off. bedroom & B & & 58.3 & 60 \\
\hline 13. & Captain bedroom & B & & 58.5 & 60 \\
\hline 14. & Captain living & B & & 57.3 & 60 \\
\hline 15. & Single cabin 4 & A & & 59.6 & 60 \\
\hline 16. & Single cabin 3 & A & & 60.2 & 60 \\
\hline 17. & Single cabin 2 & A & & 59.9 & 60 \\
\hline 18. & Single cabin 1 & A & & 58.8 & 60 \\
\hline 19. & Change room & A & & 69.2 & 90 \\
\hline 20. & Single cabin 8 & A & & 59.8 & 60 \\
\hline 21. & Single cabin 7 & A & & 62.3 & 60 \\
\hline 22. & Single cabin 6 & A & & 63.6 & 60 \\
\hline
\end{tabular}


Table 3.1 Measuring results (continue)

\begin{tabular}{|c|c|c|c|c|c|}
\hline \multicolumn{4}{|c|}{ Measuring point } & \multirow{2}{*}{$\begin{array}{l}\text { Reading } \\
{[\mathrm{dB}(\mathrm{A})]}\end{array}$} & \multirow{2}{*}{$\begin{array}{l}\text { Limit } \\
\text { [dB (A)] }\end{array}$} \\
\hline $\begin{array}{l}\text { Pos- } \\
\text { ition }\end{array}$ & Room & 总 & 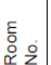 & & \\
\hline 23. & Single cabin 5 & A & & 61.1 & 60 \\
\hline 24. & Dayroom & \begin{tabular}{|l|} 
Main \\
Deck
\end{tabular} & & 69.5 & 65 \\
\hline 25. & Off. Messroom & \begin{tabular}{|l|} 
Main \\
Deck \\
\end{tabular} & & 67.5 & 65 \\
\hline 26. & Crew Messroom & \begin{tabular}{|l|} 
Main \\
Deck \\
\end{tabular} & & 71.3 & 65 \\
\hline 27. & Galley & \begin{tabular}{|l|} 
Main \\
Deck
\end{tabular} & & 69.4 & 75 \\
\hline 28. & Emergency Gener. room & \begin{tabular}{|l|} 
Main \\
Deck
\end{tabular} & & 80.3 & 90 \\
\hline 29. & Deck Office & \begin{tabular}{|l|} 
Main \\
Deck \\
\end{tabular} & & 64.3 & 65 \\
\hline 30. & Duty mess & \begin{tabular}{|l|} 
Main \\
Deck \\
\end{tabular} & & 70.2 & 65 \\
\hline 31. & Smoking room & \begin{tabular}{|l|} 
Main \\
Deck \\
\end{tabular} & & 72.1 & 65 \\
\hline 32. & Single cabin & \begin{tabular}{|l|} 
Main \\
Deck \\
\end{tabular} & & 67.9 & 60 \\
\hline 33. & Cargo Control room & \begin{tabular}{|l|} 
Main \\
Deck \\
\end{tabular} & & 73.7 & 75 \\
\hline 34. & Medical Treatment room & \begin{tabular}{|l|} 
Main \\
Deck \\
\end{tabular} & & 65.3 & 60 \\
\hline 35. & ECR & Plattorm & & 73.6 & 75 \\
\hline 36. & Between DGs & Plattorm & & 107.5 & 110 \\
\hline 37. & Steering Gear room & Plattorm & & 87.6 & 90 \\
\hline 38. & Separators room & Plattorm & & 88.7 & 90 \\
\hline 39. & $E / R$ aft side & \begin{tabular}{|c|} 
Tank \\
Top \\
\end{tabular} & & 104.2 & 110 \\
\hline 40. & $E / R$ fore side & \begin{tabular}{|c|} 
Tank \\
Top
\end{tabular} & & 106.7 & 110 \\
\hline 41. & Bowthruster room & $\begin{array}{c}\text { Under } \\
\text { MD }\end{array}$ & & 82.4 & 90 \\
\hline
\end{tabular}

The above Table no. 3.1 shows the results in every space with measured values (Reading column) and permissive values (Limit column) according to [1], SOLAS Ch. II-1, Reg. 3-12 Noise Code MSC. 337(91): CODE ON NOISE LEVELS ONBOARD SHIPS.

\section{LOCATION OF MEASURING POINTS}

Very important in analysis is choosing of space where the measurement would be performed. Therefore, should choose the places where seafarers live and work: resting cabins, messrooms, dayrooms, wheelhouse, workshop, ER, ECR, wingbridge, office, galley, cargo control room, steering gear room, etc... The location of all measuring points is shown in the below Fig. 4.1...4.7, according to [2]:

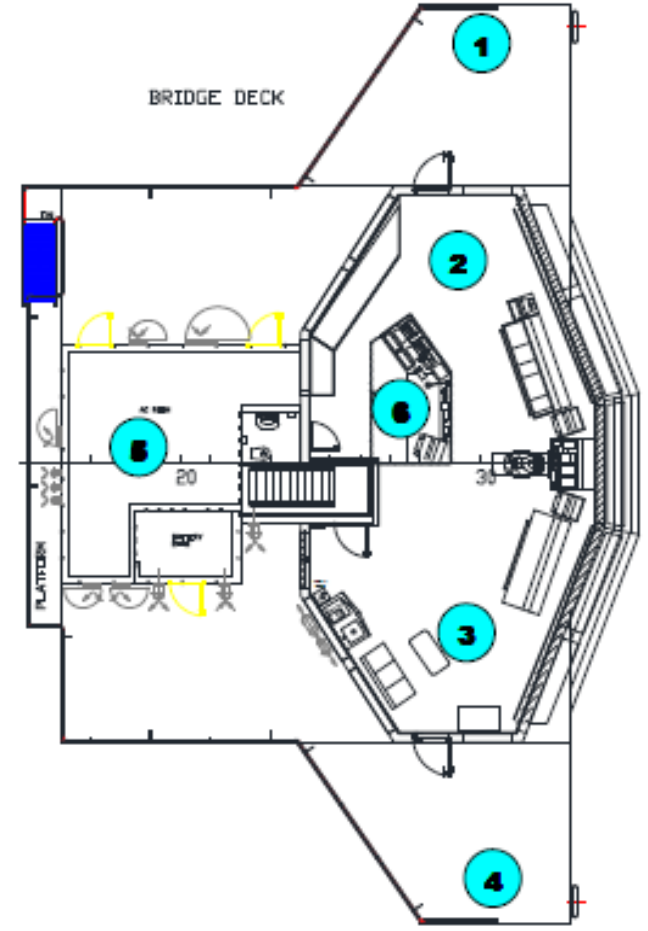

Fig. 4.1 BRIDGE Deck

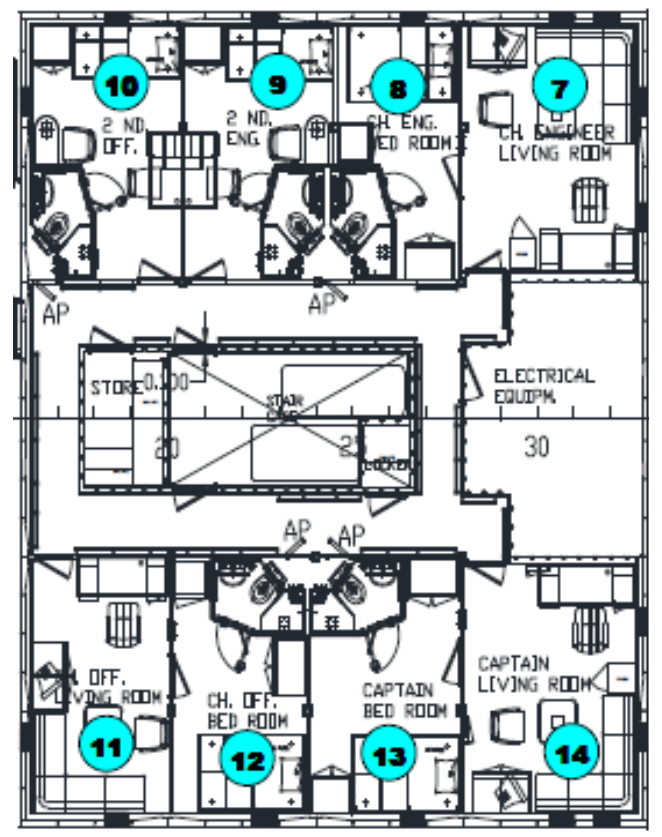

Fig. 4.2 B Deck 


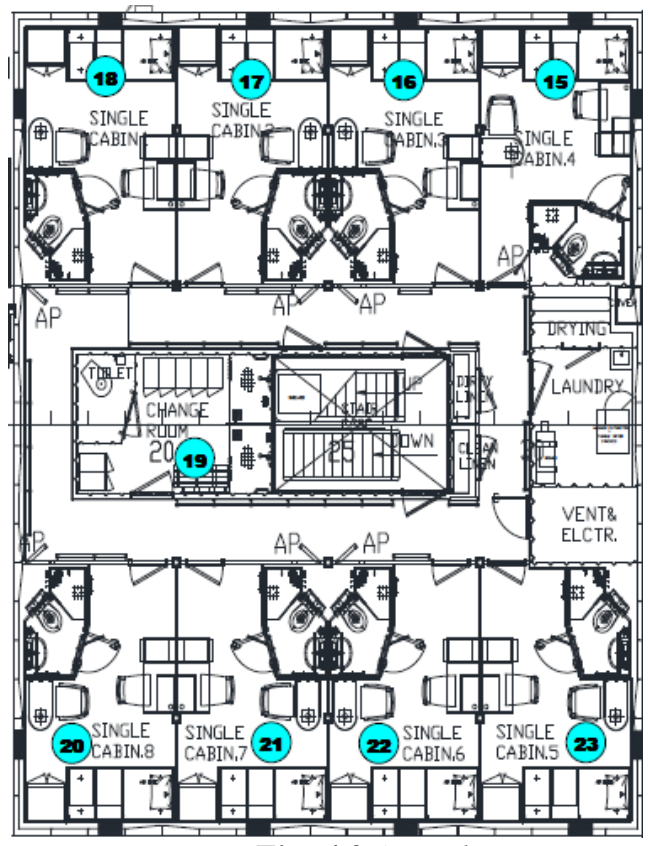

Fig. 4.3 A Deck

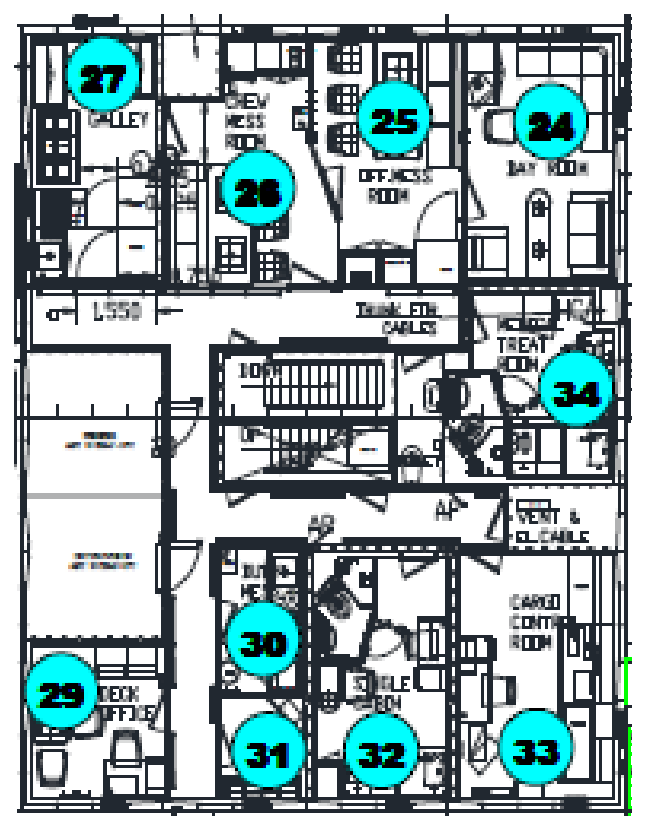

Fig. 4.4 Main Deck

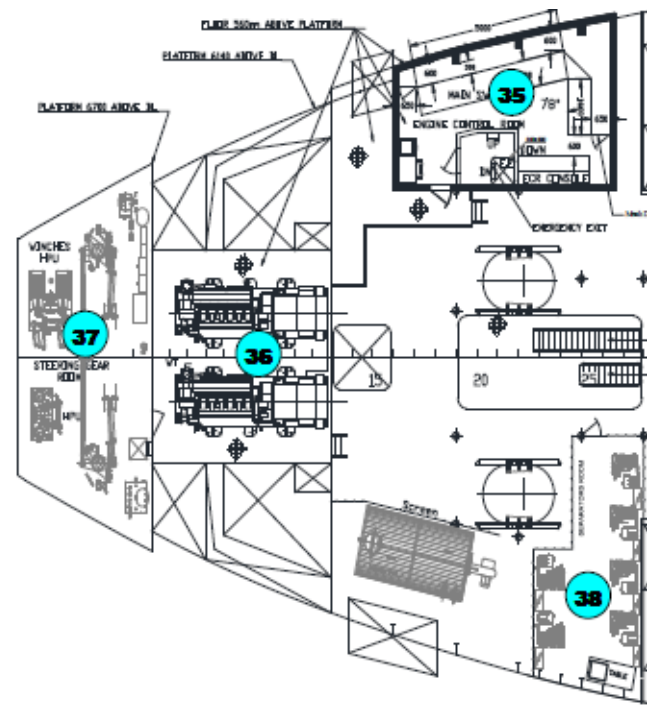

Fig. 4.5 Platform under Main Deck

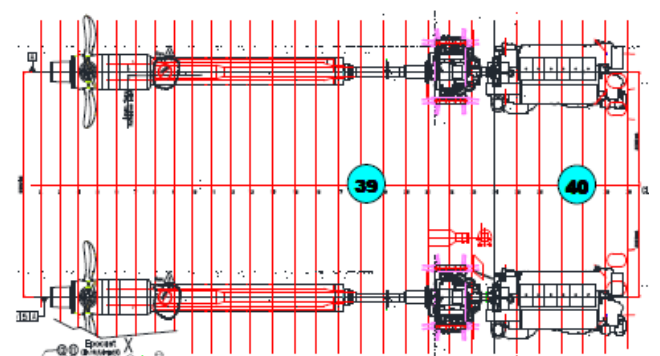

Fig. 4.6 Engine Room 


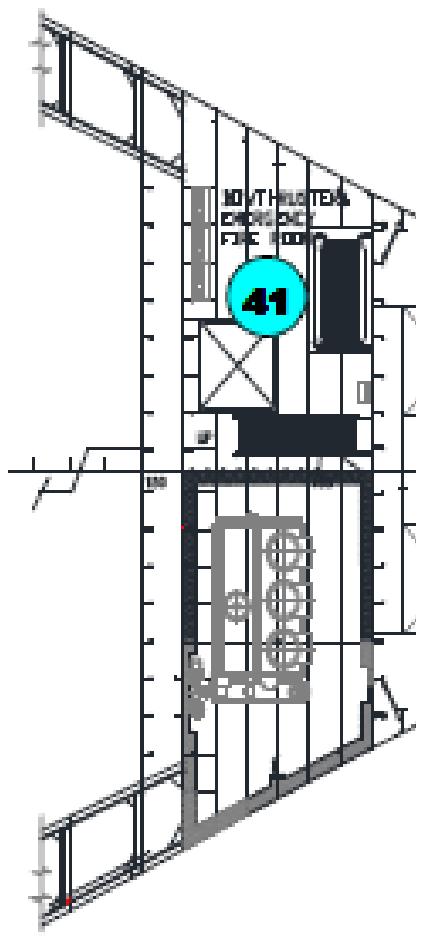

Fig. 4.7 Platform under Main Deck

The measurements have been performed with a 2250L Brüel \& Kjaer - Denmark sound level meter. Before and after measurements, the instruments have been calibrated with a NC-74 Rion - Japan acoustic calibrator, having a valid certificate.

\section{CONCLUDING REMARKS}

The structure and noise sources from this paper are similar with [3] which shows the noise levels under the limits of [1].

The analysis of measured results for this vessel (oil/chemical tanker) showed certain exceeding of limit values which can put in danger the health of seafarers:

- $\quad$ PS Wingbridge (position 1);

- Chief Officer Livingroom (position 11);

- $\quad$ Single cabin 3 (position 16);
- $\quad$ Single cabin 7 (position 21);

- $\quad$ Single cabin 6 (position 22);

- $\quad$ Single cabin 5 (position 23);

- Dayroom (position 24);

- $\quad$ Officer Dayroom (position 25);

- Crew Dayroom (position 26);

- $\quad$ Single cabin (position 32);

- Medical Treatment room (position $34)$

To fix these exceeding and to decrease the noise levels under limits of MSC 337(91), some consistent expenses was necessary for: - an additional mineral wool layer mounted in walls;

- an additional mineral wool layer mounted in ceiling;

- floating floor, etc...

All these expenses could have been avoided if a detailed analysis (a noise prediction) would have been done early, in design stage of the ship.

Because this noise prediction was missing, the designer was not able to take suitable measures to reduce noise in the above spaces. Certainly, a noise prediction would reveal in the design stage, the potential exceeding points and the cost with fixing these problems would have been much lower.

\section{Acknowledgements}

The research was supported by the Research Department of Diagnose and Measurements Group Ltd. Galati, inside the partnership with CONSTANTZA SHIPYARD SA.

The author thanks to Mr. Dipl.eng. Marius CORNEA and Mrs. Dipl. eng. Luminita BASCHIR from Constantza Shipyard SA, who made possible this research onboard a vessel built for a foreign owner. 


\section{REFERENCES}

[1]. ****Resolution MSC 337(91) "CODE ON NOISE LEVELS ONBOARD SHIPS", 2012;

[2]. ***Diagnose and Measurements Group srl Galati, "Noise Measurements Report (in accommodation) onboard Oil/Chemical Tanker $9000 \mathrm{cbm} \quad$ M/T MAYOURY", 2017;
[1]. [3] Bosoanca, I., "Noise onboard FT module carrier vessel" - The Annals of "Dunarea de Jos" University of Galati, Fascicle XI - Shipbuilding, pp. 81-84, 2017.

Paper received on December $31^{\text {th }}, 2018$ 